\title{
A New Two-Dimensional Scintillation Detector System for Small-Angle Neutron Scattering Experiments
}

\author{
G. Kemmerling, R. Engels, N. Bussmann, U. Clemens, M. Heiderich, R. Reinartz, H. Rongen, J. Schelten,
} D. Schwahn, and K. Zwoll

\begin{abstract}
A new two-dimensional position-sensitive detector system for small-angle neutron scattering experiments is under development. It is based on a $60 \times 60 \mathrm{~cm}^{2}$ large ${ }^{6} \mathrm{Li}$-glass scintillator for the neutron capture and dispersion of the emitted light on an array of photomultiplier tubes for the derivation of the event position. For subthermal neutrons of $8 \AA$, an efficiency of $96 \%$ and a spatial resolution of $8 \mathrm{~mm}$ are to be achieved. Major improvements have been made in the signal- and data-processing branch, which aims at an electronical dead time of $1 \mu \mathrm{s}$. A fast digitalization method using free-running analog-to-digital converters for each photomultiplier combined with field-programmable gate arrays for a parallel event detection in all channels has been applied. A subsequent farm of up to 16 floating-point digital signal processors is used to provide enough computing power for an improved reconstruction of the event position according to a maximum likelihood method.
\end{abstract}

\section{INTRODUCTION}

$\mathbf{S}$ MALL-angle neutron scattering (SANS) is an essential tool for the investigation of structural and dynamical properties of condensed matter. Almost any fluctuation in composition, density, or magnetization on a scale between 5-5000 A can be detected by this method [1]. Measurements are usually carried out with large-area position sensitive detectors. This allows an efficient exploitation of the expensive beam-time by a simultaneous measurement of the total scattering regime.

At future neutron facilities, like the research reactor FRM-II [2] or the European Spallation Source ESS [3], SANS will obtain a major improvement. The much higher neutron fluxes will lead to an intensity gain of at least one order of magnitude for SANS instruments, allowing a more detailed study of complex structures and fast processes. As these intensities are expected to exceed the counting capabilities of today's commonly used two-dimensional detectors, a strong emphasis on the development of new detector systems is required.

This paper describes the design of a new detector system for the SANS-diffractometer KWS1 at the research reactor FRJ-2 of the Forschungszentrum Jülich, Germany, which is carried out

Manuscript received October 13, 2000; revised February 15, 2001 and March 19, 2001

G. Kemmerling, R. Engels, N. Bussmann, U. Clemens, R. Reinartz, H. Rongen, and K. Zwoll are with the Zentrallabor für Elektronik, Forschungszentrum Jülich, Jülich D-52425, Germany (e-mail: G.Kemmerling@fz-juelich.de).

M. Heiderich and D. Schwahn are with the Institut für Festkörperforschung, Forschungszentrum Jülich, Jülich D-52425, Germany.

J. Schelten is with the Institut für Schicht-und Ionentechnik, Forschungszentrum Jülich GmbH, Jülich 52425, Germany.

Publisher Item Identifier S 0018-9499(01)06977-5.

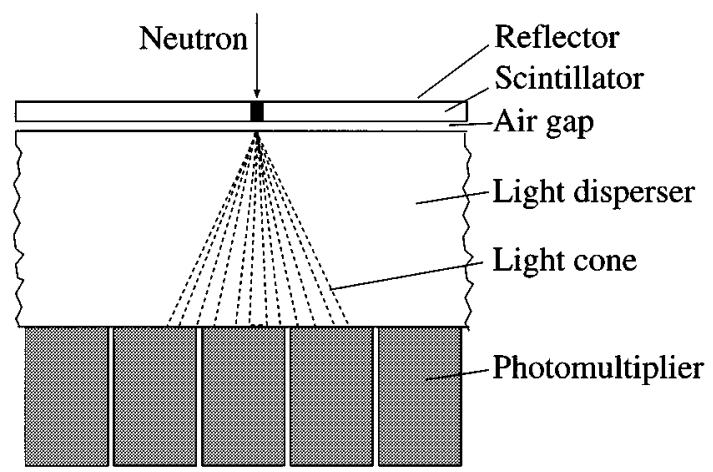

Fig. 1. Cross-sectional view of the detector showing the light distribution of a neutron event.

in the Juelicher Digital Detector Readout System project (JuDiDt). At KWS1, a position-sensitive detector [4] has been successfully used for more than 15 years now. However, after an installation of advanced neutron guides, there are now higher intensities available, leading to saturation effects of the detector at counting rates above $10 \mathrm{kHz}$. The limiting factor of this detector is the readout electronics. Due to an analog-based calculation of the neutron position, large shaping times of the analog signals were required, resulting in an electronical dead time of about $10 \mu$ s. Thus, in order to take advantage of the higher neutron intensities, the electronics branch has been completely revised for the new detector. The new design comprises a fast signal and data processing by usage of modern technologies like field-programmable gate arrays (FPGAs) and digital signal processors (DSPs). Additionally, a new reconstruction method will be used, which aims at an enlarged and more homogeneous position reconstruction. As it is envisaged that this system will be used also later at FRM-II, it must be able to cope with counting rates of several hundred kilohertz.

\section{DETECTOR}

The basic concept of the new detector front end is very similar to that of the existing KWS-1 detector and is based on the principle of an Anger camera [5] (Fig. 1). A ${ }^{6} \mathrm{Li}$-glass scintillator doped with $\mathrm{Ce}$ as an activator is used for the detection of neutrons via the nuclear reaction

$$
n+{ }^{6} \mathrm{Li} \rightarrow{ }^{3} \mathrm{H}+\alpha+4.78 \mathrm{MeV} .
$$

The excited states induced by each capture give rise to the emission of light, which is dispersed on an array of photomultiplier 


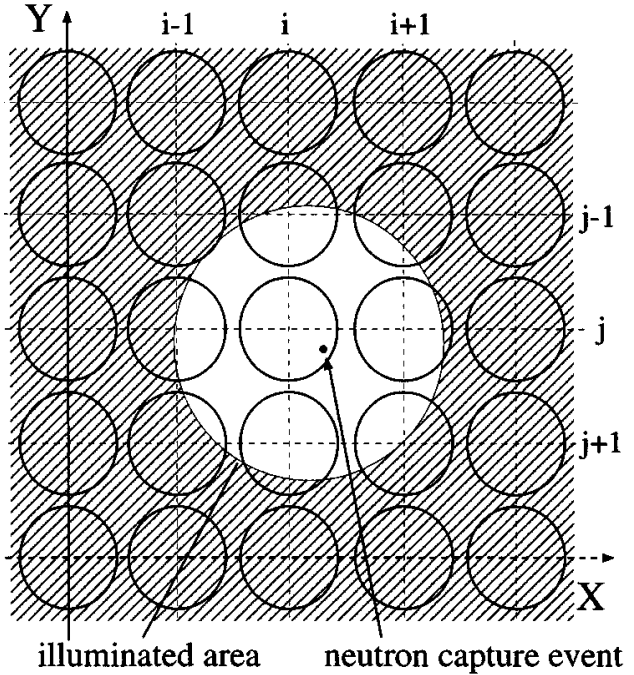

Fig. 2. Projection of the light cone from a neutron capture event on the plane of the PMT-cathodes.

tubes (PMTs). The analog signals of these PMTs are then used to derive the position of the incident neutron.

Most of the parameters for the new detector have been taken from the existing KWS-1 detector and are based on a numerical simulation [6]. Thus, the glass scintillator has a thickness of 1 $\mathrm{mm}$ and contains $17.1 \%{ }^{6} \mathrm{Li}$, resulting in an efficiency of $96 \%$ for 8 - $\AA$ neutrons. It consists of 16 plates with a dimension of $15 \times 15 \mathrm{~cm}^{2}$, which are glued with optical contact on a support glass frame, forming an active area of $60 \times 60 \mathrm{~cm}^{2}$. On the top side, the scintillator is covered with reflecting powder in order to increase the fraction of the emitted photons, which reach the photocathode of the PMTs. There are about 400 photoelectrons expected in the PMTs per neutron capture. The air gap between the scintillator and light disperser is to produce a critical angle beyond which light undergoes total internal reflection. Thus the light received by the PMTs is limited to a cone, whose diameter on the PMT-plane depends linearly on the disperser thickness.

The simulation showed that the disperser thickness influences the linearity and resolution of the detector. With increasing thickness, the linearity improves while the resolution becomes worse. The optimal thickness is $10 \%$ less than the separation of adjacent PMTs. As there is an array of $8 \times 8$ photomultiplier tubes with 3-in diameter used for light detection, the separation between adjacent PMTs has been chosen to be $83 \mathrm{~mm}$, resulting in a disperser thickness of $75 \mathrm{~mm}$. Then the light cone produced by a neutron event covers an array of nine PMTs (Fig. 2) and a spatial resolution of about $8 \mathrm{~mm}$ is to be achieved.

\section{ELECTRONICS DESIGN}

Although the new detector system will be first used at the KWS-1 diffractometer at the FRJ-2 in Jülich, it is understood to be essential that the signal- and data-processing performance be adopted for utilization at future neutron sources. In fact, there are already plans to move the whole installation later to the new FRM-II reactor in München. There, the counting rates in SANS frequently can reach the $1-\mathrm{MHz}$ range. An overall electronical dead time of about $1 \mu \mathrm{s}$ is required for the new system. A fast readout electronics with parallel signal and data processing is seen to address this problem in an appropriate way.

The electronics design is shown in Fig. 3. As mechanical assembly form, it utilizes a single 6U 19-in VME crate, which has been equipped with three partly overlapping bus systems on the backplane. There is a private bus with CompactPCI bus (CPCI) connectors, used for transfer of analog signals and board synchronization; a modified VME bus (mVME) for a fast data transfer of up to $80 \mathrm{MBytes} / \mathrm{s}$; and a standard CPCI for the communication with a host CPU. Four board types have been implemented according to the different steps in signal and data processing.

Detector signals are first amplified by preamplifiers. Each of the anodes of the 64 PMTs is connected to its preamplifier, which is mounted close to the detector. The signals are then input to the analog board, which is to filter and amplify the preamplifier signals. An analog board contains the main shaping amplifiers for 16 PMT channels. To achieve a sufficient signal-to-noise ratio, the PMT signals will be differentiated and integrated to retrieve an amplified unipolar Gaussian pulse shape with a time constant of $200 \mathrm{~ns}$.

Signals are then fed to the ADC/FPGA board, which has the task of signal digitalization and event triggering. Because there are four boards of this type in the system, their clocks have to be synchronized in order to avoid electronical time displacements. This is done via the private bus. An ADC/FPGA board contains free-running 10-bit ADCs for 16 channels and an FPGA (XILINX XCV300-BG432) with a local first-in first-out (FIFO) of $4 \mathrm{k}^{*} 36$ bit for data buffering. During measurements, the ADCs permanently digitize the signals with a clock rate of $20 \mathrm{MHz}$ and deliver their values to the FPGA. Then the FPGA performs a parallel peak detection on the basis of a sliding mean value determination [7], whereas a detected peak means a valid event. Inside the FPGA, discriminators have been implemented. By checking the crossing of lower levels at the beginning of the pulse and the falling below such a level at the end of the pulse, they should largely reduce the misinterpretation of noise and the occurrence of pileup effects. Once a peak has been recognized, a signal is emitted to the mVME bus on which all FPGAs on different boards write their current values into the FIFO. Up to 800 events can be buffered in the FIFOs, which allows for an averaging of load distribution. Programming of the FPGA can be performed via a CAN bus interface, which allows for a switching between different operating modes.

For further data processing, two types of DSP boards with mixed VME and CPCI interfaces are used. The first type, the local bus controller (LBC), contains a single DSP (TMS320 C6201), which is to control the data flow. On the second board type, there are four floating-point DSPs (TMS320 C6701), which are to be used for the reconstruction of the event position. Up to four of such boards may be inserted in the system in order to provide sufficient computing power for position reconstruction. In case of a valid event, the LBC gets the signal from the corresponding FPGA, looks up a free DSP, and instructs it to read the data out of the FPGA FIFO-buffers via the modified VME bus. There, the position of the event is calculated and the belonging histogramming memory position is incremented. Accumulated data may then be accessed from the host CPU. 


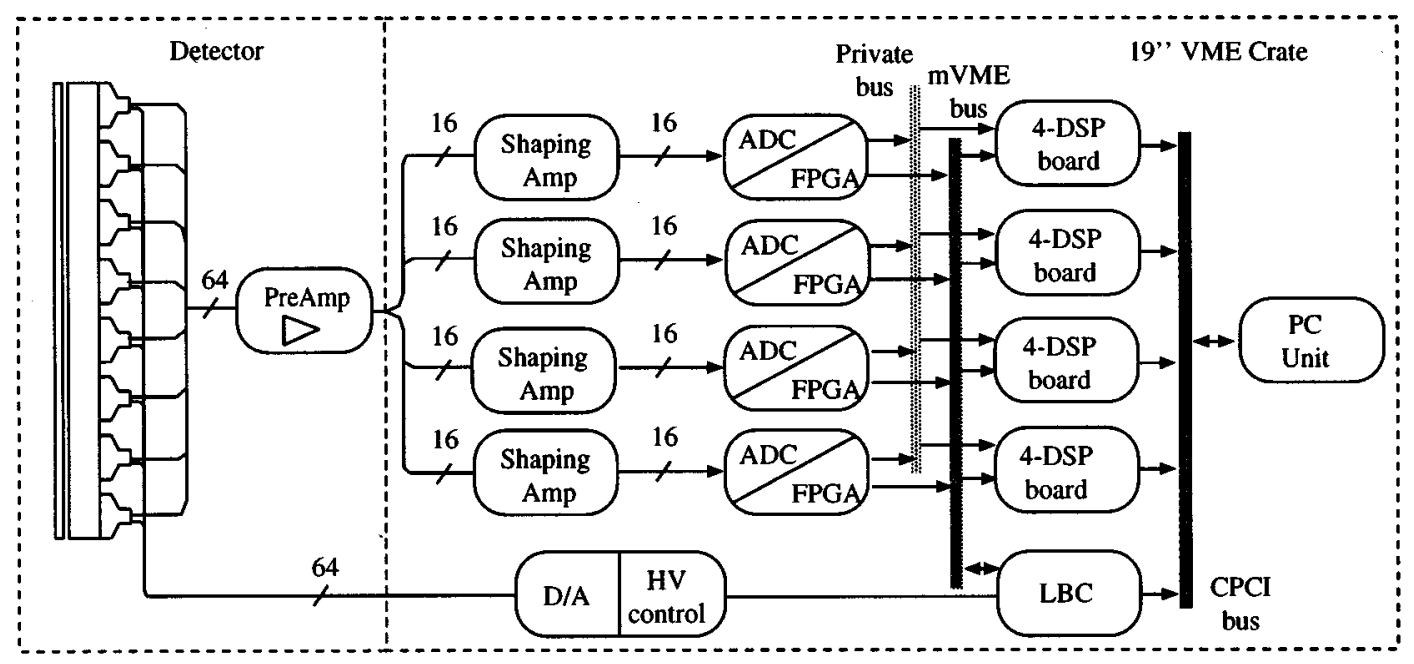

Fig. 3. Block diagram of the analog and digital signal processing.

\section{POSITION RECONSTRUCTION}

In the reconstruction, the position of the neutron event is calculated from the 64 PMT signals. But that is only possible in a restricted way, because the PMT signals fluctuate stochastically around their expectation values and are therefore not a definite function of the position. Custom methods, like center-of-gravity (COG) determination, show for detectors of this type of construction strong inhomogeneities, which are not tolerable for a precise reconstruction of the position. Fig. 4 shows the result of a detector simulation, which created a uniform distribution of events over the whole detector area. Especially at the edges of the detector and in the middle between the PMTs there are considerable nonlinearities, which might be corrected.

To avoid such shortcomings, a new reconstruction method has been derived. It is based on a lookup of the event position with the maximum likelihood, which is described by the probability sum

$$
(x, y):=\operatorname{Max}\left[P(x, y)=\prod_{j=1}^{64} P_{j}\left(S_{j}, E_{j}(x, y)\right)\right]
$$

where $P_{j}$ denotes the probability of finding the PMT signal $S_{j}$, which has the expectation value $E_{j} . P_{j}$ are Poisson distributions with a peak behavior at $S_{j}=E_{j}$. They may be described in good approximation by Gaussian functions with $\left(S_{j}-E_{j}\right)$ as arguments. Instead of a calculation of the maximum value for $P(x, y)$, it is then sufficient to find the minimum of the weighted sums of the square deviations. This is a similar approach as used in least square fits. The minimum deviation $D$ is then defined by

$$
D=\sum_{i=0}^{9} \frac{\left(S_{i}-\gamma E_{i}\right)^{2}}{2 E_{i}}
$$

where only those PMTs are considered, which are covered by the light cone (see Fig. 2). The scale factor $\gamma$ has been introduced to ensure that only the relative values are considered in the minimum search. It reflects the fraction of the signal sum to the sum of expectation values and has to be computed for each query. To restrict the search on a limited query, there are only discrete event positions permitted. For an expected resolution

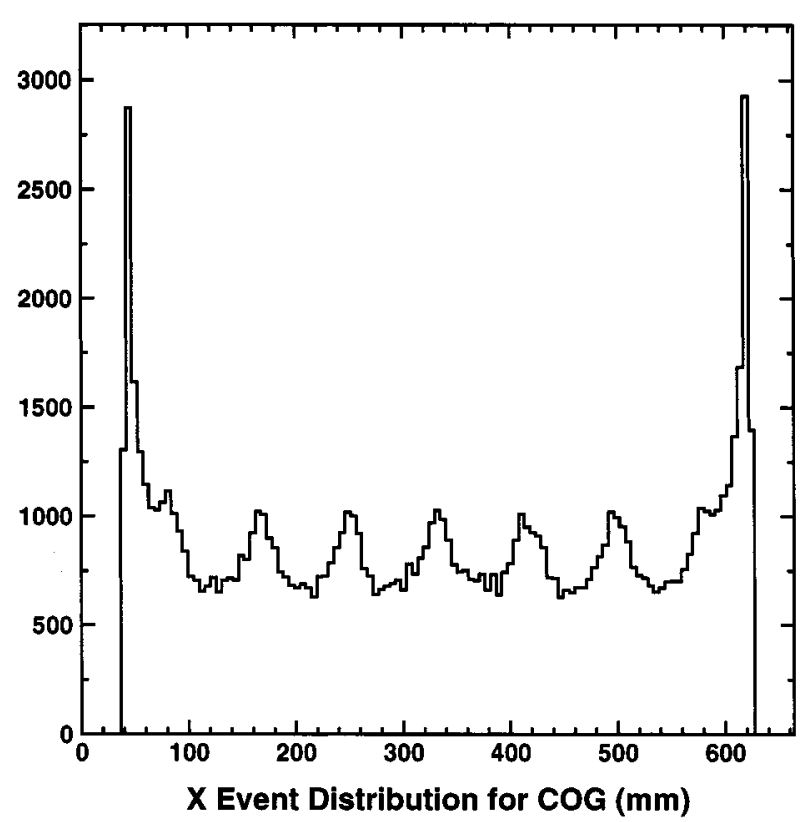

Fig. 4. Reconstruction results with center-of-gravity method.

of $8 \mathrm{~mm}$, a division of the whole detector into 128 channels in each dimension seems to be appropriate. The expectation values of the PMT signals for each of these channels can then be either calculated by a simulation or measured by a step-wise movement of a neutron source over the whole detector.

In the DSP, the position reconstruction is carried out by a two-step process. At first, the coarse address of the event is determined by finding the box of nine photomultipliers with the highest signal amount and the subsequent calculation of the center-of-gravity position. The corresponding channel then serves as the starting point for the maximum-likelihood search. In an iteration, the deviations of the neighboring channels are calculated and the channel with the smallest value serves as the starting point for the next iteration. The procedure stops either by finding the minimum value or by reaching the maximum value of allowed iteration, which has to be introduced due to the maximum time limit. Fig. 5 shows the result of a simulation, carried out with the same data as above for the COG method. 


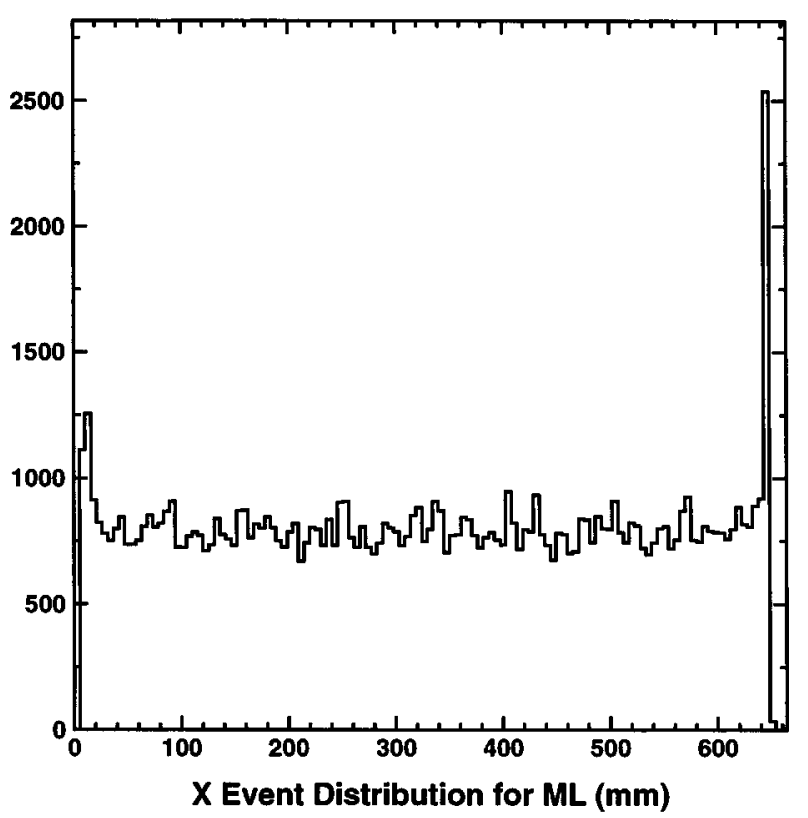

Fig. 5. Reconstruction results with maximum-likelihood method.

By comparison of the two results, it is obvious that the maximum-likelihood method delivers a more homogeneous detector response than the COG calculation. The nonlinearities of the COG position determination, which are due to undetected photons in the space between the PMTs, disappear in the maximum-likelihood reconstruction because they are considered by the expectation values. This is particularly remarkable at the detector edges. While COG has a reconstruction limit in the middle of the outermost PMTs, the maximum-likelihood method performs a linear reconstruction nearly up to the detector edges.

\section{BACKGROUND SUPPRESSION}

Background radiation has to be considered for large-area neutron detectors. One source is the high-energy cosmic radiation, which sums up to about $12-14 \mathrm{~cm}^{-2} \cdot \mathrm{h}^{-1}$ at the existing KWS-1 detector. Another source is the usually large $\gamma$-background, originating directly from the reactor or from activated material in the surrounding. It has an energy spectrum in the $\mathrm{MeV}$ range with a strong intensity increase at lower energies. Particularly at measurements with weak scattering samples, this background spoils the results because of the sensitivity of the ${ }^{6} \mathrm{Li}$-scintillator for this radiation. Therefore, it is mandatory to suppress it as far as possible.

For the new detector system, background suppression is done in two steps. At first, the large amount of lower energy $\gamma \mathrm{s}$ as well as the high-energy cosmic radiation is rejected by applying appropriate lower and upper discriminator levels inside the FPGAs during peak detection. The second step is then carried out in the reconstruction during COG finding. By calculation of the pulseheight sum of an event, one can accurately determine events whose pulse heights are outside the range of the neutron peak. Thus, in the very first step of reconstruction, one can filter out those events that do not belong to the neutron peak. This should lead to a $\gamma$-ratio on the order of $10^{-4}$ per neutron.

\section{PMT STABILIZATION}

A long-term stability of the PMT gains is necessary for the previously described reconstruction method. As it is known that the gain of PMTs may drift in time with a rate on the order of percents per week, a stabilization unit is foreseen for the system (see Fig. 3). It utilizes a gain adjustment by changing the PMT high voltage with a digital-to-analog converter.

For this purpose, pulse-height spectra are simultaneously taken for each PMT during measurements. Care must be taken because of the Gaussian form of the light intensity on the PMT plane. To obtain narrow spectra, only the highest PMT signal value of an event is inserted, which corresponds to the PMT next to the event. After measurements, one can then obtain a matching of average pulse heights of all PMTs by changing their high voltages.

\section{CONCLUSION}

The design of a new position-sensitive detector system for small-angle neutron scattering experiments has been described. It is based on a scintillation detector equipped with modern readout electronics. The strength of the design is a fast parallel signal- and data-processing by usage of FPGA and DSP technologies. It is expected to cope with intensities at future neutron facilities. Furthermore, a new reconstruction algorithm has been implemented, which is based on a maximum likelihood calculation of the event position. As simulation shows, it will improve the position reconstruction by delivering a more homogeneous detector response than commonly used methods.

The detector system is currently in the construction phase. PMTs, disperser, and scintillator arrived, and their local inhomogenities have been surveyed for later calibration purposes. Mounting of these components in the mechanical frame has also been finished. In electronics, first prototype boards have been designed and manufactured. They are now in the test phase. The desired algorithm for position reconstruction has been ported to the DSP platform and needs currently a computation time of about $20 \mu \mathrm{s}$. This is already close to the required reconstruction speed but still needs some optimization. Commissioning of the whole system is envisaged in the middle of 2001.

\section{REFERENCES}

[1] J. Schelten and R. W. Hendrichs, "Recent developments in X-ray and neutron small-angle scattering instrumentation and data analysis," $J$. Appl. Cryst., vol. 11, pp. 297-324, 1978.

[2] W. Gläaser and W. Petry, "The new neutron source FRM II," Physica B, vol. 276-278, pp. 30-32, 2000.

[3] R. Wagner, W. Bräautigam, D. Filges, and H. Ullmaier, "The project 'European Spallation Neutron Source (ESS)': Status of R\&D programme," Physica B, vol. 276-278, pp. 38-44, 2000.

[4] M. Heiderich, R. Reinartz, R. Kurz, and J. Schelten, "A two dimensional scintillation detector for small angle neutron scattering," Nucl. Instrum. Meth., vol. A 305, pp. 423-432, 1991.

[5] H. O. Anger, "Scintillation camera," Rev. Sci. Instrum., vol. 29, pp. 27-33, 1958.

[6] J. Schelten and R. Kurz, "Computer modeling of position-sensitive scintillation detectors," in Proceedings of the Conference on Position-Sensitive Detection of Thermal Neutrons, P. Convert and J. B. Forsyth, Eds. London, U.K.: Academic, 1983, p. 203.

[7] P. J. Pietraski, Z. Zojceski, D. P. Siddons, G. C. Smith, and B. Yu, "Digital centroid-finding electronics for high-rate detectors," IEEE Trans. Nucl. Sci., vol. 46, no. 4, pp. 810-816, 1999. 